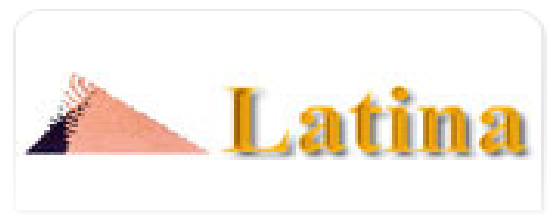

Revista Latina de Comunicación Social

E-ISSN: 1138-5820

jpablos@ull.es

Laboratorio de Tecnologías de la Información y Nuevos Análisis de Comunicación Social España

Valdez Zepeda, Andrés; Huerta Franco, Delia A.; Aguilar Aldrete, Arturo; Pérez, Octavio Adolfo El voto de los mexicanos en el extranjero. Un análisis de los resultados de 2006, a la luz de las teorías sobre el comportamiento político

Revista Latina de Comunicación Social, vol. 10, núm. 62, enero-diciembre, 2007, p. 0 Laboratorio de Tecnologías de la Información y Nuevos Análisis de Comunicación Social

Canarias, España

Disponible en: http://www.redalyc.org/articulo.oa?id=81906218

Cómo citar el artículo

- Número completo

- Más información del artículo

Página de la revista en redalyc.org

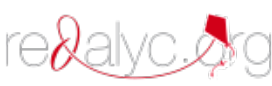

Sistema de Información Científica

Red de Revistas Científicas de América Latina, el Caribe, España y Portugal Proyecto académico sin fines de lucro, desarrollado bajo la iniciativa de acceso abierto 


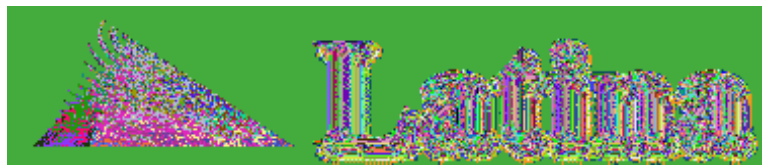

Revista Latina de Comunicación Social 62 enero - diciembre de 2007

Edita: LAboratorio de Tecnologías de la Información y Nuevos Análisis de Comunicación Social Depósito Legal: TF-135-98 / ISSN: 1138-5820

Año 10 - 2a época - Director: Dr. José Manuel de Pablos Coello, catedrático de Periodismo

Facultad de Ciencias de la Información: Pirámide del Campus de Guajara - Universidad de La Laguna 38200 La Laguna (Tenerife, Canarias; España) Teléfonos: (34) 922317231 / 41 - Fax: (34) 922317254

Investigación

FORMA DE CITAR ESTE TRABAJO EN BIBLIOGRAFÍAS, SEGÚN LA APA:

Valdez Zepeda, Andrés; Huerta Franco, Delia A.; Aguilar Aldrete, Arturo; Pérez Preciado, Octavio Adolfo (2007). El voto de los mexicanos en el extranjero. Un análisis de los resultados de 2006, a la luz de las teorías sobre el comportamiento político. Revista Latina de Comunicación Social, 62. Recuperado el x de xxx de 200x de: http://www.ull.es/publicaciones/latina/200718Valdez_Huerta_Aguilar_Perez.htm

[Revisora / revisor: Creo que el artículo "El voto de los mexicanos en el extranjero. Un análisis de los resultados de 2006, a la luz de las teorías sobre el comportamiento político" trata una asunto de interés y actualidad, de trascendencia histórica para México, con rigor y claridad y una perspectiva científica digna de mención. El debate se sitúa en una de las fronteras de referencia mundial de flujos migratorios, México/USA, y también de desigualdad social; por éstas y otras razones creo que es un artículo del máximo interés para Revista Latina de Comunicación Social contar con la mencionada investigación. Respecto a los encuestados, ciudadanos mexicanos con derecho a voto y residentes en el extranjero, creo que este estudio es de interés extraordinario por ser un trabajo de campo original y sobre el que se sustenta la tesis del artículo, creo que conviene resaltar el valor de este trabajo de campo, excelente desde el punto de vista cualitativo y muy expresivo de una experiencia inicial y poco conocida.]

\title{
El voto de los mexicanos en el extranjero. Un análisis de los resultados de 2006, a la luz de las teorías sobre el comportamiento político
}

\section{The Vote of the Mexican Immigrants. An Analysis of the Results of the 2006 Presidential Election, to the light of political behavior theories}

\author{
Artículo recibido el 19 de julio de 2007 \\ Sometido a pre-revisión (Comité de Redacción) el 20 de julio de 2007 \\ Enviado a revisión el 23 de julio de 2007 \\ Devuelto a autor tras primera revisión el 23 de julio de 2007 \\ Recibido de autor tras primera revisión el 29 de julio de 2007 \\ Aceptado el 22 de agosto de 2007 \\ Galeradas telemáticas a disposición del autor el 23 de agosto de 2007 \\ Visto bueno del autor, el 26 de agosto de 2007 \\ Publicado el 27 de agosto de 2007
}

Dr. Andrés Valdez Zepeda @ [C.V.]

Catedrático

Universidad de Guadalajara, UdG, Jalisco, México

azepeda@cucea.udg.mx

Mgt. Delia A. Huerta Franco @ [C.V.]

Profesora investigadora

Universidad de Guadalajara, UdG, Jalisco, México

deliaahf@cucea.udg.mx

Mgt. Arturo Aguilar Aldrete @ [C.V.]

Investigador

Universidad de Guadalajara, UdG, Jalisco, México.

Mgt. Octavio Adolfo Pérez Preciado @ [C.V.]

Investigador

Universidad de Guadalajara, UdG, Jalisco, México.

ocpere@cucea.udg.mx

Resumen: El 2 de julio de 2006, por primera vez en la historia del país, los mexicanos residentes en el extranjero pudieron emitir su voto en una elección presidencial. De acuerdo a los resultados dados por el Instituto Federal Electoral (IFE), Felipe Calderón, candidato del Partido Acción Nacional (PAN) de orientación derechista, fue el que obtuvo el mayor número de estos sufragios sequido por Andrés Manuel López obrador candidato de la Alianza por el Bien de Todos de orientación 
electorales a la luz de las diferentes teorías sobre el comportamiento del voto de los emigrantes. Se concluye que el voto de los mexicanos en el extranjero reproduce y refleja tanto la forma en la que votan los emigrantes en su lugar natal, como la influencia política y cultural que ejerce en la conducta del votante el sistema político y electoral del nuevo país de residencial.

Palabras clave: Voto de los mexicanos en el extranjero - elecciones presidenciales de 2006 - México - conducta del elector - candidatos y partidos políticos - teorías políticas - Felipe Calderón - López Obrador - Instituto Federal Electoral (IFE).

Abstract: The 2 of July of the 2006, the Mexican residents abroad could for the first time voted in a presidential election. According to the results given by the electoral federal institute (IFE), Felipe Calderon, candidate by the National Action Party (PAN) of right oriented, was the one that obtained the majority of these suffrages, followed by Andres Manuel Lopez Obrador, candidate of the "alliance for the good of the people," of leftist orientation. These was the most competitive election ever take place in Mexico. In the essay, electoral results of this election are analyzed to the light of different theories about the voting behavior of the emigrants. We concludes the vote of the Mexicans abroad reproduces and reflects so much the way in which the emigrants in their native place vote, as well as the political and cultural influence that exerts the conduct of the voter the political and electoral system of the new country of residence.

Key Words: Mexican voters abroad - presidential elections 2006 - political theories - candidates and political parties voting behaviour - Felipe Calderón - López Obrador - Instituto Federal Electoral (IFE).

Sumario: 1 Introducción. 2. Enfoques teóricos. 3. Los supuestos sobre la inclinación del voto. 4. Desinterés partidista. 5. Conclusiones. 6. Bibliografía. 7. Notas.

Summary: 1. Introduction. 2. Teoretical perspectives. 3. Hypothesis about the voting behaviour. 4. Political party apathy 5. Conclutions. 6. Bibliography. 7. Notes.

\section{Introducción}

El derecho al voto de los residentes en el extranjero, cuyos antecedentes más añejos los encontramos en el Reino Unido desde 1951 y que hoy día se ha extendido, al menos, a 64 naciones del orbe (Navarro, 2005) [1], se ha considerado dentro de la teoría política como parte, por un lado, de la ampliación de los derechos políticos de los ciudadanos y, por el otro, como parte del proceso de desarrollo y consolidación de los sistemas democráticos [2]. De esta forma, se aduce que sólo se vota cuando hay elecciones y las elecciones, organizadas sobre una base de libertad y legalidad, forman parte de los sistemas democráticos, considerando que la dotación de este derecho a los ciudadanos que han salido, voluntaria u obligatoriamente, de su país de origen, por diferentes motivos, forman parte del propio desarrollo de las democracias modernas (Valdés, 2005) [3].

En el caso de México, este derecho se hizo efectivo en la pasada elección presidencial del 2 de julio de 2006 [4], aunque sólo se pudo votar por vía postal de acuerdo con lo que marca la normatividad electoral y después de cubrir una serie de requerimientos y tramites burocráticos, que desmotivaron enormemente la participación ciudadana [5]. De esta forma, emitieron su sufragio sólo 32, 261 electores [6] de un padrón estimado por el Instituto Federal Electoral (IFE) de 4.2 millones de posibles votantes [7], en una de las regiones de inmigración de referencia mundial (México/Estados Unidos de Norteamérica) en materia tanto de flujos migratorios como de desigualdad social.

Ahora bien, los resultados dados a conocer por el IFE, señalan que Felipe Calderón obtuvo el 57.42 por ciento de los votos, seguido por Andrés Manuel López Obrador, quien alcanzó el 33.43 por ciento. [8]. El candidato de la Alianza por México, Roberto Madrazo, obtuvo el 4.1 por ciento de los sufragios, mientras que Patricia Mercado, del Partido Alternativa, alcanzó el 2.68 por ciento y Roberto Campa, del Partido Nueva Alianza, el 0.39 por ciento de los votos, como se muestra en el cuadro No. 1. [9]

\section{Cuadro No. 1}

\begin{tabular}{|c|c|c|c|c|c|}
\hline Candidato & Partido o Coalición & $\begin{array}{l}\text { Número de } \\
\text { Votos en el } \\
\text { Extranjero* }\end{array}$ & $\begin{array}{c}\% \text { de } \\
\text { Votación } \\
\text { validad }\end{array}$ & $\begin{array}{l}\text { Número de } \\
\text { Votos en } \\
\text { México }\end{array}$ & $\begin{array}{c}\% \text { de } \\
\text { Votación }\end{array}$ \\
\hline $\begin{array}{l}\text { Andrés } \\
\text { Manuel López } \\
\text { Obrador }\end{array}$ & $\begin{array}{c}\text { Alianza por el Bien } \\
\text { de Todos }\end{array}$ & 11,088 & 33.43 & $14,756,350$ & 35.31 \\
\hline $\begin{array}{l}\text { Roberto } \\
\text { Madazo } \\
\text { Pintado } \\
\end{array}$ & Alianza por México & 1,360 & 4.1 & $9,301,441$ & 22.26 \\
\hline $\begin{array}{l}\text { Felipe } \\
\text { Calderón } \\
\text { Hinojosa }\end{array}$ & $\begin{array}{l}\text { Partido Acción } \\
\text { Nacional }\end{array}$ & 19,016 & 57.42 & $15,000,284$ & 35.89 \\
\hline $\begin{array}{l}\text { Roberto } \\
\text { Campa }\end{array}$ & $\begin{array}{l}\text { Partido Nueva } \\
\text { Alianza }\end{array}$ & 128 & 0.39 & 401,804 & 0.95 \\
\hline $\begin{array}{l}\text { Patricia } \\
\text { Mercado }\end{array}$ & $\begin{array}{c}\text { Alternativa } \\
\text { Socialdemócrata y } \\
\text { Campesina }\end{array}$ & 887 & 2.73 & $1,128,850$ & 2.70 \\
\hline
\end{tabular}

Fuente: Instituto Federal Electoral, IFE. * No se incluyen los votos nulos. 
durante el proceso electoral en materia de proselitismo político? En el presente escrito se analiza, a la luz de las diferentes teorías sobre el voto y el comportamiento político de los residentes en el extranjero, el caso de la pasada elección presidencial de 2006, tratando de dar respuesta a estas interrogantes.

Para la realización de la presente investigación se realizó una revisión hemerográfica en los diarios de circulación nacional en México durante el periodo previo y posterior de la elección presidencial, así como de las plataformas programáticas de los diferentes partidos políticos de México [10].

\section{Los enfoques teóricos}

Las teorías sobre el comportamiento político de los emigrantes en el extranjero y, en lo particular, sobre su comportamiento electoral, apuntan sobre tres posibles conductas. La primera señala que el comportamiento, las actitudes, los valores y las opiniones políticas de los residentes en el extranjero son muy similares a las predominantes en su lugar de origen. Esto es, la cultura política y la preferencia electoral de los emigrantes guarda una correspondencia con la existente en su lugar de origen (Calderón y Martínez, 2002), ya que lo que se presenta es una especie de espejo de lo que pasa en el país de procedencia. De esta forma, por ejemplo, si su núcleo de origen, familia y comunidad en México tiende mayoritariamente a votar por un determinado partido, esa conducta electoral se repetirá por los emigrantes en el extranjero. En este sentido, la estancia y experiencia de los mexicanos en el extranjero no genera un cambio en la conducta y comportamiento electoral de los emigrantes. Esto implica que no hay una real integración cultural y, de cierta manera, política al nuevo país de residencia y a su paradigma político (Huntington, 2004).

Esta teoría, si bien explica algunos patrones culturales y políticos de las comunidades migrantes no considera que se genera ciertos cambios en el comportamiento político de las comunidades de origen del migrante, durante el tiempo que éstos se encuentran fuera del país. Esto es, varias comunidades y núcleos de población de origen del migrante han experimentado, con el tiempo, ciertas transformaciones, como la alternancia en el gobierno y cambios en la conducta y lealtad electoral, lo que repercute también en su cultura política [11]. Además, este enfoque teórico descarta toda posibilidad de influencia del paradigma político predominante en el nuevo país de residencia del emigrante, lo cual resulta poco creíble si lo analizamos en una perspectiva de largo plazo, principalmente con los individuos que radican en el exterior por largos períodos de tiempo.

La segunda teoría, contrapuesta a la primera, apunta que los migrantes experimentan un proceso de socialización política en el nuevo país de residencia que los hace cambiar de actitudes, valores y, en lo general, de cultura política, lográndose una real integración y apropiación no sólo cultural, sino también respecto del paradigma político predominante, de tal forma que si ellos radican en un país con un sistema político democrático, tenderán a adoptar pautas de conducta propias de un sistema democrático (DeSipio, 1996). En este mismo sentido, los emigrantes en el extranjero tenderán a inclinar su voto a favor de la opción política que más se ajuste al paradigma político e ideológico que predomina en su nuevo lugar de residencia (De la Garza, 1996).

Esta teoría, si bien aporta algunos elementos para un mejor entendimiento del comportamiento electoral del emigrante, deja de lado todo el aspecto cultural, inercial y de hábito de votación de los electores que han adquirido en su país de origen. Sin embargo, estudios recientes sobre el comportamiento electoral apuntan que tanto la inercia (el hábito y costumbre de votación que se ha formado a través de los años), así como la circunstancia (la nueva realidad y coyuntura política que se vive en el nuevo país de residencia) influyen en la decisión final del elector (De la Heras, 1999).

A partir de estas dos concepciones teóricas, podemos desarrollar una tercera teoría, de orientación ecléctica, la cual apunta que los emigrantes en el extranjero modifican su cultura política en parte, pero que también conservan varios rasgos y actitudes propios de la cultura política y hábitos de votación predominantes en su lugar de origen. En este sentido, esta orientación teórica señala que sí existe un impacto e integración con la cultura predominante en el nuevo país de residencia [12], pero también existen patrones culturales propios de su lugar de origen que generan una nueva cultura política. Esto es, los emigrantes no se asimilan totalmente a la cultura política del nuevo país de residencia, aunque sí toman algunos patrones y marcos de referencia que incide en su comportamiento político, pero siguen conservando ciertos valores, actitudes y conductas propias de su país de origen. Entonces, lo que realmente se genera es una nueva subcultura política híbrida en los emigrantes, que bien podríamos llamar 'cultura política trasfronteriza' [13].

\section{Los supuestos sobre la inclinación del voto}

Antes de los comicios del 2 de julio, no existía la seguridad de que el voto de los residentes en el extranjero beneficiara a un determinado partido y, en consecuencia, a su candidato presidencial [14]. Sin embargo, fue común encontrar varios comentarios, muchos de estos incluso contradictorios, de algunos analistas y estudiosos de los fenómenos políticos, tratando de explicar la posible orientación y el comportamiento del voto de los mexicanos en el extranjero. Al respecto, lo único que se pudo platear, a priori, fueron sólo supuestos, ya que, previo a la elección, no se realizaron estudios de opinión serios sobre las preferencias electorales que pudieran ser concluyentes y que nos pudieran haber dado una mejor idea y acercamiento sobre las intenciones y preferencias partidistas de los emigrantes.

De esta forma, en vísperas de la elección se debatió sobre la existencia de tres diferentes supuestos o escenarios sobre la posible inclinación del voto a favor de las tres principales fuerzas partidistas de México, quienes se disputaron férreamente la presidencia de la república en una de las elección más competidas de la historia política de México. A continuación, se describe esta tríada de supuestos, que no fueron excluyentes entre sí y que representaron los escenarios más posibles en la campaña presidencial de 2006.

\section{a. Supuestos a favor de la izquierda}

Uno de los supuestos más referidos afirmó que los votos de los mexicanos residentes en el extranjero serían de oposición tanto al PRI como al PAN, ya que, según señalaban, por su culpa, en la medida que ambos han sido gobierno, fueron obligados a salir del país en búsqueda de empleo y mejores oportunidades de vida que no encontraron en México. De esta forma, los apoyadores de este supuesto apuntaban que la Alianza por el Bien de Todos sería la que mayor beneficio electoral obtendría de este sector de votantes, ya que ni el PRI ni el PAN, cuando han sido gobierno, han podido reducir los niveles de pobreza y marginación ni han mejorado las condiciones económicas del país, lo que pudiera haber incidido en una 
Este supuesto, señaló, además, que el voto de los mexicanos en el extranjero sería mayoritariamente de izquierda, lo que favorecerá al PRD, ya que tradicionalmente los mexicanos en los Estados Unidos han apoyado a los candidatos del Partido Demócrata, que son más "progresistas" y se han opuesto a los candidatos del Partido Republicano que son más conservadores [15]. Es decir, este supuesto consideró que existe una preferencia e inclinación ideológica de los mexicanos en el extranjero que favorecía a la Alianza por el Bien de Todos y que imposibilitaba el apoyo a los otros partidos (PRI y PAN), por ser mucho más conservadores.

Finalmente, este supuesto aducía que el PRD (la Alianza por el Bien de Todos) era el partido que enarbolaba la bandera de los pobres y muchos connacionales, que hoy día viven en el extranjero, son de origen humilde, lo que tenderían a apoyar a su candidato.

\section{b. Supuestos a favor del PRI}

Existieron diversas voces dentro del sector de prí́stas que señalaba que el voto de los mexicanos en el extranjero beneficiaría a su partido y a su candidato presidencial, por varias razones. Primero, apuntaban que el mayor porcentaje de emigrantes en el extranjero son de origen rural, predominando un hábito de votación y una cultura política que los favorecía. En esta misma línea de argumentación, señalaba que el nivel escolar de los emigrantes es generalmente bajo y que, tradicionalmente, los sectores con menor escolaridad en México tienden a votar por este partido. De esta forma, la mayoría de los votos de los mexicanos en el extranjero serían, decían ellos, para la Alianza por México, integrada por el PRI y el Partido Verde Ecologista de México.

Este supuesto, afirmaba, además que por su longevidad, el PRI (fundado en 1929) y en menor medida el PAN (fundado en 1939), son los dos partidos más conocidos por los emigrantes, ya que el PRD (fundado en 1989), por su juventud, es realmente poco conocido en el extranjero, lo que representaría una ventaja competitiva en la contienda electoral a favor de Roberto Madrazo Pintado.

Solamente algunos prístas, por cierto los menos, reconocían que muchos de los votos de los emigrantes mexicanos serían sufragios de protesta, que no sólo afectarían al PRI sino también al PAN, reconociendo que serían perjudicados, pero señalando que esta carga la compartirían con el Partido Acción Nacional.

\section{c. Supuestos a favor de la derecha}

El último gran supuesto señalaba que el voto de los emigrantes sería un sufragio de protesta antipriísta porque por su culpa tuvieron que salir del país en búsqueda de empleo y mejores oportunidades de vida que no encontraron en México, y que si bien el PAN hoy es gobierno por un lustro o quinquenio, la mayor culpa la tiene el PRI, que gobernó más de 70 años el país, por lo que la "factura" realmente la tendrían que pagar los prí́stas.

Este supuesto aducía, además, que los sufragios favorecerían al PAN por ser durante este gobierno foxista, que se permitió el voto de los mexicanos en el extranjero, ya que durante los gobiernos prí́stas nunca prosperó este derecho, a pesar de que era una vieja demanda de los emigrantes [16].

Este supuesto, también, apuntaba que los emigrantes han palpado un verdadero cambio cuando regresan a México para visitar a sus familiares y seres queridos, pudiendo constatar que ya no hay tanta corrupción y que el Programa Paisano [17] cambió radicalmente la forma en que son tratados los connacionales al llegar y transitar por el país, lo que contrasta con lo que sucedía en el pasado. Finalmente, este supuesto consideraba que la imagen del presidente Vicente Fox Quesada es muy buena en el extranjero, lo que influiría en la orientación del voto de los emigrantes mexicanos.

En suma, estos tres grandes supuestos trataban de dar una explicación a la posible orientación del voto de los mexicanos residentes en el extranjero, apuntando concretamente al partido, alianza o candidato que pudiera haberse beneficiado con dicho sufragio y la razón del apoyo.

\section{Preferencias electorales}

Con el fin de conocer las preferencias electorales de los mexicanos residentes en el extranjero y poder cuantificar los posibles apoyos que cada uno de los candidatos y partidos podrían obtener de estos sufragios, se realizó un sondeo, vía telefónica, en agosto de 2005 [18], a 320 mexicanos residentes en los Estados Unidos sobre su interés en participar en las elecciones en México reveló que la mayoría (un 60\%) desconocía su nuevo derecho de votar, la mayoría no tenía interés en participar en las elecciones presidenciales de 2006 (70\%) y de los que manifestaron interés en participar (30\%), la inclinación de su voto estaba dividida mayoritariamente en dos preferencias: el PAN (43\%) y el PRD (40\%). El PRI sólo obtenía el $14 \%$ de las simpatías electorales. El resto (3\%) no manifestó su inclinación partidista.

Este sondeo, de cierta manera, concedió con el resultado final de la elección, ya que los electores residentes en el extranjero se inclinaron a votar mayoritariamente por el candidato del PAN, aunque de manera más amplia, como se muestra en el siguiente cuadro.

\section{Comparativo entre el sondeo y los resultados definitivos}

\begin{tabular}{|l||c||c||c|}
\hline \multicolumn{1}{|c|}{ Partido } & Sondeo 2005 & Resultados de la votación en el extranjero (\%) & Diferencia (\%) \\
\hline \hline PAN & $43 \%$ & 58.54 & 15.54 \\
\hline \hline Alianza por el Bien de Todos & $40 \%$ & 34.13 & 6.13 \\
\hline \hline Alianza por México & $14 \%$ & 4.18 & 9.92 \\
\hline \hline Sin simpatía & $3 \%$ & --- & --- \\
\hline
\end{tabular}




\section{Desinterés partidista}

De acuerdo a lo establecido por la normatividad electoral, no fue posible hacer campaña en el extranjero por los candidatos ni por los partidos políticos. Además, el voto sólo se pudo efectuar vía postal, descartándose otras opciones como el voto presencial y el voto electrónico [19]. Para que los emigrantes se informaran de este nuevo derecho político, así como de los tiempos y mecanismos de la elección presidencial de 2006, el IFE, Instituto Federal Electoral, realizó una campaña de difusión en medios impresos y electrónicos mucho antes de la elección (desde agosto de 2005 a enero de 2006), lo que incluyó informar sobre los mecanismos a seguir para emitir su voto [20].

Sin embargo, debido al bajo número de ciudadanos que se inscribieron en el padrón de votantes $(40,854)$, prácticamente los candidatos y partidos no realizaron esfuerzo alguno por tratar de ganar este sufragio, enfocando sus esfuerzos principalmente a obtener los votos en México. Es decir, en la medida que la cifra de emigrantes empadronados fue muy baja, el interés de los partidos por obtener este voto también fue baja [21].

Al final, como se comentó, los electores residentes en el extranjero se inclinaron mayoritariamente a favor del candidato del PAN, Felipe Calderón, quien obtuvo un 57.42 por ciento de los votos, seguido por Andrés Manuel López Obrador, quien logró un 33.43 por ciento de los sufragios.

\section{A manera de conclusión}

El derecho al voto de los residentes en el extranjero ha sido considerado como parte de los procesos de desarrollo democrático y, en lo particular, como parte de la ampliación de los derechos políticos de los connacionales, quienes por diferentes motivos han tenido que emigrar a otra nación. Este es un derecho existente en México desde 1996, cuando se reformó la Constitución General de la República, mismo que entró en vigencia a partir de las pasadas elecciones presidenciales.

La teoría política sobre el comportamiento electoral de los ciudadanos residentes en el extranjero apunta que son tres los tipos de comportamiento principales que se generan entre estos electores. El primero, que bien podríamos denominar como 'la teoría del espejo', aduce que la conducta del emigrante es un reflejo de lo que pasa en su comunidad de origen. La segunda, que podríamos denominar 'la teoría del imán' [22], señala que el sistema y cultura política predominante en el nuevo país de residencia logra trasformar las actitudes, cosmovisión y conducta política de los residentes en el extranjero, de tal forma que éstos logran adoptar como suya la nueva cultura política predominante en el país de residencia. Finalmente, la última teoría, que aquí denominamos como 'yuxtapuesta', apunta que lo que realmente se crea es una nueva cultura híbrida, que integra tanto aspectos de la vieja como de la nueva cultura política que predomina tanto en el país de origen como en el de la nueva residencia del emigrante. A la luz de estas teorías es cómo debemos entender el comportamiento político de los mexicanos residentes en el extranjero, en la pasada elección presidencial.

De acuerdo a los resultados de la elección dados a conoce por el IFE, el caso mexicano reafirma la pertinencia de estas tres teorías sobre el voto de los emigrantes. Por un lado, los votantes se inclinaron mayoritariamente por Felipe Calderón, reafirmando, de cierta manera, 'la teoría del espejo', que apunta que los electores tienden a votar de la misma manera como lo hacen sus connacionales en su país de origen. Es decir, como pasó en México, el voto mayoritario favoreció al candidato del PAN [23].

Sin embargo, este resultado no puede ser entendido sin recurrir a lo que hemos denominado como 'teoría del imán', la cual apunta que las transformaciones culturales y la influencia política a la que están expuestos los emigrantes en el nuevo lugar de residencia influyen en su comportamiento político. Esto es, los emigrantes mexicanos que viven en su mayoría en los Estados Unidos decidieron optar mayoritariamente por el PAN, quien representa, de cierta manera, una opción conservadora muy similar a las opciones políticas que existen en la Unión Americana.

De esta manera y en concordancia con 'la teoría yuxtapuesta', la inclinación del sufragio estuvo influenciada tanto por lo que pasa en el país de origen como por el tipo de sistema político predominante en el nuevo lugar de residencia de la mayoría de los emigrantes mexicanos.

Finalmente, de acuerdo a los datos de las autoridades electorales, el candidato de la Alianza por el Bien de Todos, Andrés Manuel López Obrador, obtuvo un segundo lugar en la votación, lo cual confirma ambas teorías (espejo e imán) sobre el comportamiento del voto de los emigrantes. El bajo porcentaje de votación a favor de Madrazo (4.1\%) confirma la teoría yuxtapuesta antes señalada, la cual refleja no sólo un rechazo de los emigrantes al candidato presidencial, sino además una afrenta a las políticas gubernamentales y las acciones que por muchos años el PRI impulsó en México y que generaron una alta migración.

\section{Bibliografía}

1. CALDERON Chelius Leticia y Martínez Saldaña Jesús, La dimensión política de la emigración mexicana, Instituto Mora, México, 2002.

2. DESIPIO Louis, Counting on the Latino Vote: Latinos as a New Electorate, Charlottesville, VA, University Press of Virginia, 1996.

3. DE LAS HERAS, María, "Uso y abuso de las encuestas. Elección 2000: los escenarios", en la colección Con una cierta mirada, de Editorial Océano, 1999.

4. DE LA GARZA, Rodolfo, "Will the Real Americans Please Stand Up: Anglo and Mexican-American Support of Core American Political Values", en American Journal of Political Science, 1996, 40. No. 2, pp. 335-51. 
Valdez Zepeda, Andrés; Huerta Franco, Delia A.; Aguilar Aldrete, Arturo; Pérez Preciado, Octavio Adolfo (2007).... Página 6 de 7

Periódicos nacionales mexicanos

El Universal, Ciudad de México, consultas entre enero-julio de 2006

Reforma, Ciudad de México, febrero julio de 2006.

La Jornada, Ciudad de México, enero - julio de 2006.

Milenio, Ciudad de México, enero - agosto de 2006.

\section{Notas}

[1] En América Latina se permite el voto de los residentes en el extranjero en El Salvador, Colombia, Venezuela, Brasil, Paraguay, Chile, República Dominicana, Honduras, Argentina y Perú. De estas 64 naciones, en 27 de ellas se permite el voto postal, aunque solamente Puerto Rico pertenece a la región de América Latina y el Caribe.

[2] Otros países se han preocupado no sólo por otorgar el derecho al voto de los residentes en el extranjero, sino también en poner límites en este derecho. Por ejemplo, este derecho al voto de los residentes en el extranjero se pierde en Canadá después de 5 años interrumpidos de estar fuera del país, en Australia después de 6 años y en Gran Bretaña después de 15 años.

[3] Conferencia impartida por Leonardo Valdés Zurita en el Departamento de Estudios Internacionales de la Universidad de Guadalajara, 31 de agosto de 2005, como parte del foro "El voto de los mexicanos en el extranjero".

[4] La publicación del decreto que garantizaba este derecho al sufragio para los connacionales que viven en el extranjero se dio el día 30 de junio de 2005, haciendo con esto efectivo las reformas constitucionales de 1996 que ya contemplaban dicho derecho, pero del cual no existía la legislación reglamentaria.

[5] Sin embargo, de acuerdo a este decreto, las campañas de proselitismo político fueron prohibidas, pudiendo votar únicamente aquellos ciudadanos que contaban con credencial del IFE, hubieran solicitado su participación antes del 15 de febrero de 2006 y hubieran mandado sus papeles (copia de su credencial electoral y un comprobante de domicilio) a las oficinas de la autoridad electoral en México. Además, de acuerdo a este mismo, los interesados deberían haber dado aviso por escrito al IFE de su intención de votar decreto antes del 15 de enero de 2006. Para consultar la normatividad electoral de México, acceda a la página Web www.ife.org.mx.

[6] Las causas del alto abstencionismo tiene que ver no sólo con el desinterés de los emigrantes para participar en la política, sino también por los limitaciones que la misma reforma electoral impuso al voto en el extranjero.

[7] En la elección de 2006, participó el 58.55 por ciento de los ciudadanos empadronados en México, presentándose una abstención del 41.45 por ciento, una de las más grandes de los últimos años.

[8] López Obrador fue candidato por la Alianza por el Bien de Todos integrada por el Partido de la Revolución Democrática (PRD), el Partido del Trabajo (PT) y el Partido Convergencia (PC).

[9] La votación promedio de sufragios de emigrantes en otros países es menor al 10 por ciento del padrón estimado.

[10] Las plataformas programáticas de los partidos políticos fueron consultadas en las páginas electrónicas siguientes, entre enero y julio del 2006: www.pri.org.mx, www.pan.org.mx y www.prd.org.mx.

[11] Sobre esto, surgen algunas interrogantes. Por ejemplo, ¿qué impacto tienen este tipo de cambios culturales en la conducta política del migrante? ¿De qué manera los cambios en la comunidad de origen del emigrante transforman e inciden en su cultura política?

[12] Principalmente de aquellos que tienen muchos años viviendo en el extranjero, aunque ocasionalmente regresen a su país de origen.

[13] Esta nueva subcultura la podríamos definir también con la frase "ni de aquí ni de allá," en la medida que no es exactamente un reflejo de las culturas políticas predominantes en los países tanto de origen como de recepción de la emigración.

[14] No hay estudios serios y actuales sobre el comportamiento de los mexicanos en el extranjero. Como antecedentes sólo ubicamos dos aproximaciones. La primera en 1981 en los Estados Unidos, donde se realizó un sondeo por Jorge Bustamante, entonces investigador del Colegio de la Frontera Norte, el cual reveló que el 47 por ciento de los mexicanos votarían en contra del PRI. La segunda experiencia se dio en las elecciones de 1988 y 1994, cuando se realizaron elecciones en algunos estados de la unión americana de carácter simbólico paralelas a las realizadas en México. El resultado: no más del 17 por ciento de los mexicanos votaron por el PRI y su candidato. La oposición se llevó la mayoría de los sufragios.

[15] Aquí es importante decir que el Partido Republicano ha avanzado enormemente en captar el voto de los hispanos, como se reflejó en la última elección presidencial donde fue reelecto George W. Bush como presidente de los Estados Unidos de Norteamérica, de tal forma que hoy nadie puede asegurar que el voto hispano es sólo de impronta demócrata.

[16] Las primeras demandas para permitir el voto de los mexicanos en el extranjero se dio después del movimiento revolucionario de 1917-21. Al inicio de la década de los cuarenta y en el marco del convenio migratorio entre los Estados Unidos y México que legalizaba la contratación de emigrante mexicanos, también se demandó que se posibilitará el voto de los mexicanos en los Estados Unidos de Norteamérica. 
Valdez Zepeda, Andrés; Huerta Franco, Delia A.; Aguilar Aldrete, Arturo; Pérez Preciado, Octavio Adolfo (2007).... Página 7 de 7

[17] Este programa busca atender las necesidades de los emigrantes y facilitar el viaje de los mexicanos que provienen de los EEUU, mejorando los servicios migratorios y de aduana, tratando de hacer confortable su estancia en el país.

[18] Este sondeo fue realizado en el estado de California, Nuevo México y Hawai entre el 15 y 30 de agosto de 2005. El sondeo realizado fue vía telefónica.

[19] Para el caso de México, el término 'normatividad electoral' se usa como sinónimo de 'normativa electoral' o 'legislación electoral'.

[20] Aquí es importante señalar que otorgar el derecho del sufragio a los ciudadanos radicados fuera del país ha sido una tendencia mundial en los últimos años, ya que, al menos, en 64 naciones se permite este tipo de sufragio, ya sea de manera directa en urnas instaladas en embajadas, consulados y en diferentes sitios públicos, o mediante votos postales o incluso, mediante sufragios emitidos bajo formatos electrónicos. Este tipo de fenómenos forma parte del proceso de ampliación de los derechos políticos de los ciudadanos y de desarrollo de la democracia liberal, pero también como producto del consenso de las diferentes fuerzas partidistas que compiten por los espacios de poder público y, sobre todo, porque el monto y porcentaje de sufragios no ha sido determinante en el resultado final de la elección. Sin embargo, debido a los grandes flujos migratorios, principalmente de los países del llamado tercer mundo hacia las naciones desarrolladas y por el monto de sufragios que pueden, en algunos casos, ser decisivos para el resultado final de la elección, empezarán a existir voces opositoras y cuestionamientos a este tipo de participaciones. Las fuerzas políticas partidistas que perciban que el voto de los ciudadanos residentes en el extranjero les es adverso serán las que con más determinación y fuerza se opondrán a que estos sufragios se sigan permitiendo en próximos procesos electorales.

[21] Para promover y organizar el voto de los mexicanos en el extranjero el IFE solicitó un presupuesto cercano a los $\$ 1,200$ millones de pesos, equivalente a un poco más de los 100 millones de dólares. Al final, sólo se ejerció cerca del 60 por ciento de este presupuesto (723 millones de pesos).

[22] De esta forma, tanto los partidos como los candidatos desaprovecharon la gran oportunidad de ver incrementado el número de votos a favor de su causa política, ya que si hubieran promovido el voto y organizado a los electores con seguridad la participación ciudadana se hubiera incrementado significativamente.

[23] El imán tiene la propiedad no sólo de atraer, sino también de trasmitir sus efectos (fuerzas magnéticas) a otros objetos metálicos, de tal forma que estos actúan al estar en contacto con el imán también como imanes atrayendo a otros objetos metálicos.

[24] De acuerdo con los resultados del IFE, Felipe Calderón se impuso por el 0.58 por ciento de los votos por encima de López Obrador. Sin embargo, estos resultados fueron impugnados por la Alianza por el Bien de Todos ante el Tribunal Federal del Poder Judicial de la Federación (TRIFE), exigiendo un recuento de votos. Al final, el TRIFE ratificó, después de un conteo parcial de los votos, el triunfo de Calderón, a pesar de las impugnaciones, lo que acrecentó el conflicto postelectoral.

FORMA DE CITAR ESTE TRABAJO EN BIBLIOGRAFÍAS, SEGÚN LA APA:

Valdez Zepeda, Andrés; Huerta Franco, Delia A.; Aguilar Aldrete, Arturo; Pérez Preciado, Octavio Adolfo (2007). El voto de los mexicanos en el extranjero. Un análisis de los resultados de 2006, a la luz de las teorías sobre el comportamiento político. Revista Latina de Comunicación Social, 62. La Laguna (Tenerife).

Recuperado el x de xxxx de 200x de:

http://www.ull.es/publicaciones/latina/200718Valdez_Huerta_Aguilar_Perez.htm 